\title{
Endometrioid Adenocarcinoma Arising From Adenomyosis in a Premenopausal Woman: A Case Report
}

\author{
Naoyo Nishida ${ }^{a, c}$, Fumihiro Murakami ${ }^{b}$, Koichi Higaki ${ }^{a}$
}

\begin{abstract}
Endometrioid adenocarcinoma derived from adenomyosis is rare. A premenopausal woman, aged 36, was referred to us for evaluation of multiple solid tumors in the pelvis. MRI revealed multiple tumors in uterine myometrium and omentum. Their T2 and enhanced T1weighted imaging led us to a diagnosis of uterine sarcoma. Hysterectomy with bilateral salpingo-oophorectomy, omentectomy, and pelvic lymphadenectomy was performed. Histopathology revealed grade 3 endometrioid adenocarcinoma with squamous differentiation. Cancer nests were observed adjacent to the adenomyotic foci. Postoperative classification was pT3aN1M1. Rejecting adjuvant therapy, the patient died 16 days later from rapid enlargement and rupture of the metastasized liver and respiratory failure. An autopsy revealed mass lesions in the pelvic cavity and compressed small intestines causing ileus. Bone metastasis, multiple lymph node metastases including para-aortic, and lung metastases were also revealed. Multiple liver metastases had caused biliary obstruction and hepatic failure. The tumor-induced hypercoagulable condition resulted in multiple thromboses, which in turn led to hemorrhaging in the lungs and eventual respiratory failure. Histological examination of the pelvic mass lesions revealed mainly poorly differentiated carcinoma. The tumor cells contained bizarre nuclei and exhibited high mitotic activity. This case was high-potential malignant tumor as the tumor rapidly grew and infiltrated multiple organs in a short period. It was a type II carcinoma with biologically aggressive behavior.
\end{abstract}

Keywords: Adenomyosis; Endometrioid adenocarcinoma; Type II endometrial carcinoma; Autopsy

\footnotetext{
Manuscript accepted for publication October 28, 2015

aDepartment of Pathology, St Mary's Hospital, 622 Tsubuku-honmachi, Kurume City, Fukuoka 830-8543, Japan

bDepartment of Obstetrics and Gynecology, St. Mary's Hospital, 622 Tsubuku-honmachi, Kurume City, Fukuoka 830-8543, Japan

'Corresponding Authir: Naoyo Nishida, Department of Pathology, St Mary's Hospital, 622 Tsubuku-honmachi, Kurume City, Fukuoka 830-8543, Japan. Email: naoji@med.kurume-u.ac.jp
}

doi: http://dx.doi.org/10.14740/jmc2357e

\section{Introduction}

Two types of endometrial carcinoma, type I and type II, have been distinguished on the basis of clinicopathological and genetic studies. Malignant transformation of adenomyosis has been recognized similar pathway of them. Type I, characterized by ER-positiveness, accounts for the majority of endometrial cancers [1]. Type II, consisting of rare cases and negative for ER and PR, is characterized by poorly differentiated adenocarcinoma. We report here an autopsy case of type II endometrial carcinoma arising from adenomyosis.

\section{Case Report}

A 36-year-old premenopausal woman with a 4-year history of adenomyosis, gravida 0 , para 0 , presenting with continued low abdominal pain, visited a local clinic. Ultrasound scans revealed multiple tumors in the pelvic cavity. On referral to our hospital, biopsy of endometrium was done, which revealed almost normal glandular cells (negative). MRI revealed multiple tumors in uterine myometrium and omentum. The tumors were enhanced on T1-weighted imaging, and uterine sarcoma was suspected. The endometrium, without enhancement, was $6 \mathrm{~mm}$ in thickness. Liver and bone metastasis was confirmed by CT (Fig. 1). Serum levels of CEA and CA125 were elevated to $695.3 \mathrm{U} / \mathrm{mL}$ and $300.0 \mathrm{U} / \mathrm{mL}$, respectively. The patient underwent hysterectomy with bilateral salpingo-oophorectomy, omentectomy, and pelvic lymphadenectomy. The uterus was child-head sized and multiple disseminations in omentum, peritoneal cavity, and adnexa were seen (Fig. 2). The cut surface of the uterus was white to yellow-white in color. Necrosis and hemorrhage were observed. Histopathological examination revealed grade 3 endometrioid adenocarcinoma with squamous differentiation. But the lesion was located only in the muscle layer of the uterus and the endometrium was no tumorous change. Cancer nests adjacent to the adenomyotic foci were observed. Tumor cells stained positively for p53, but did not express either ER or PR (Fig. 3). We diagnosed the case as a stage 4B endometrioid adenocarcinoma originating from adenomyosis, following the staging of the International Federation of Gynecology and Obstetrics (FIGO) 2008. Its postoperative classification was pT3aN1M1. 

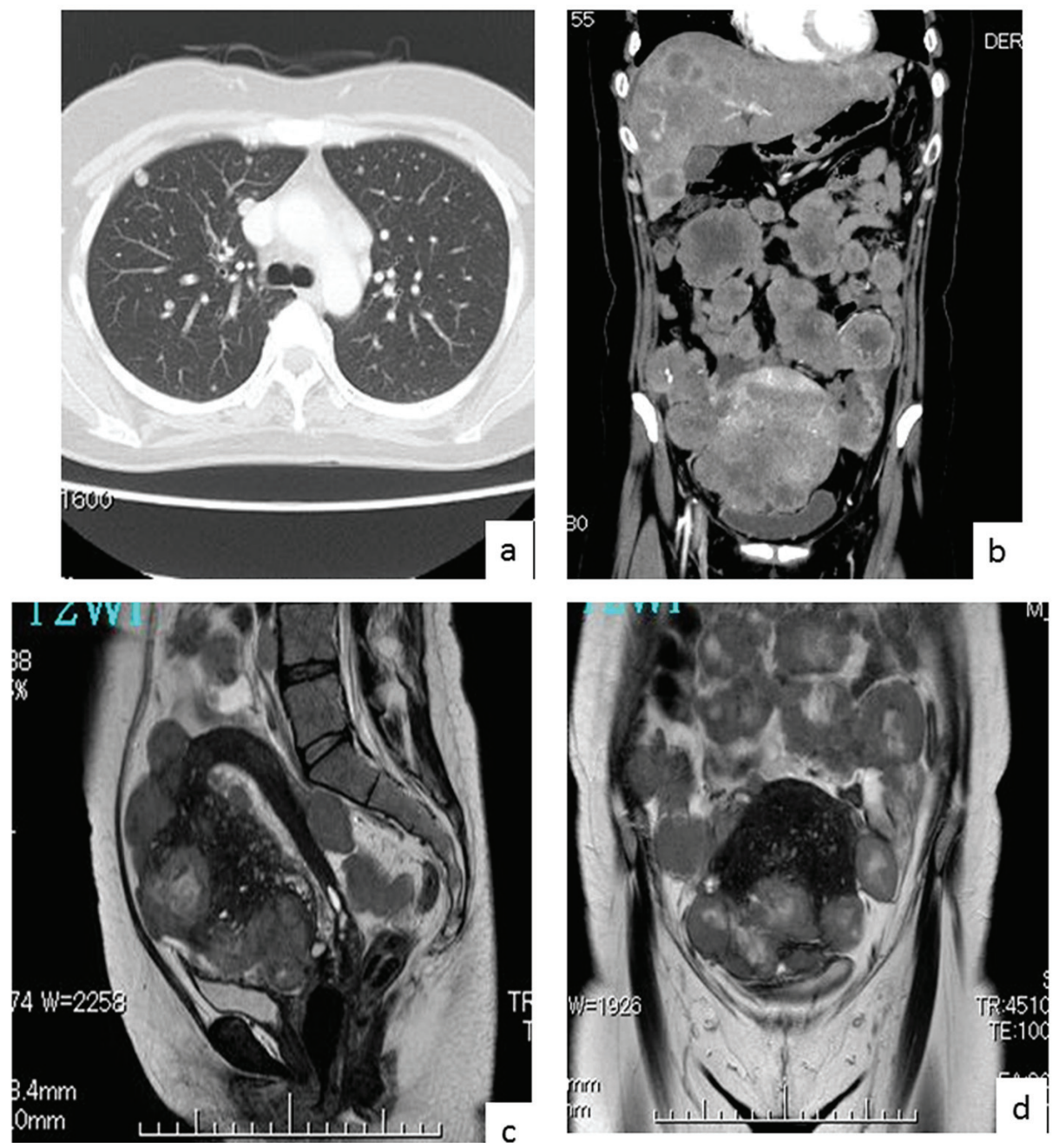

Figure 1. CT revealed multiple lung (a) and liver metastasis (b). MRI of the pelvis before admission. Sagittal (c) and frontal (d) T2-weighted image demonstrates multiple tumors in uterine myometrium and omentum.

Opting against adjuvant therapy, the patient died 16 days later from rapid enlargement and rupture of the metastasized liver and respiratory failure. An autopsy confirmed the mass lesions in the pelvic cavity and also compressed small intestines, which caused ileus. Bone metastasis, multiple lymph node metastases including para-aortic, and lung metastases were also diagnosed. Multiple liver metastases caused biliary obstruction and hepatic failure. Multiple thromboses and hemorrhaging in both lungs with the hypercoagulable condition led to respiratory failure. Histology of the pelvic mass lesion revealed mainly poorly differentiated carcinoma. The tumor cells contained bizarre nuclei and exhibited high mitotic activity. Benign endometriosis lesions were not present in pelvic cavity.

\section{Discussion}

Adenomyosis is a common gynecological disease, which is characterized by the presence of ectopic endometrial glands and stroma located within the myometrium [2]. Several pathological theories have been proposed: epithelial-mesenchymal transition [3, 4], metaplasia [5], Mullerian rests [6], reaction (injury and regeneration of tissue) $[7,8]$, and multipotential perivascular theory $[9,10]$. The etiology and pathogenesis of adenomyosis remain undecided. There have been 44 reported cases of adenocarcinoma developing within adenomyosis [11]. Most of the patients were postmenopausal. Malignant transformation of adenomyosis in premenopausal women with normal endometrium is extremely rare [12].

Sampson's or Colman's criteria for the diagnosis of carcinoma arising from adenomyosis are as follows: 1) the carcinoma must not be situated in the endometrium or elsewhere in the pelvis; 2) the carcinoma must be seen to arise from the epithelium of adenomyosis and not to have invaded from other source; and 3) endometrial (adenomyotic) stromal cells should be surrounding the aberrant glands to support the diagnosis of adenomyosis $[13,14]$. Histological subtypes of the tumors are endometrioid and, to a lesser degree, serous, clear cell and poorly differentiated adenocarcinoma $[12,15]$. Two types of 



Figure 2. Macroscopic findings of the uterus (a, b), omentum (c), and liver (autopsy) (d). The uterus was child-head sized with multiple nodular lesions and the endometrium appeared normal $(a, b)$. Multiple disseminations in omentum (c) and liver (d) were seen.

endometrial carcinoma, type I and type II, have been described on the basis of clinicopathological and genetic studies. In most cases with malignant transformation of adenomyosis, different stages of atypical or hyperplastic changes are simultaneously identified [1]. This observation points to a similar pathway of carcinogenesis in adenomyosis as is known in estrogenresponsive endometrial cancer type I [1].

In general, malignant transformation of adenomyosis is positive for ER, PR, cyclooxygenase-2 (COX-2), CA125 and focally weak-positive for aromatase. Hormonal receptor expression is associated with low-grade and early-stage tumors [11]. Kucera et al, having researched the clinical data of 219 patients with the diagnosis of early endometrial cancer, reported that adenomyosis was found in 88 of them and malignant changes in adenomyosis were present in six (6.8\%) of those 88 patients. All those six cases were with endometrioid adenocarcinoma, of which five were well or moderately differentiated. They suggested that there is a similar pathway of carcinogenesis in adenomyosis in that it is positive for ER (type I endometrial cancer) [1]. By contrast, poorly differentiated adenocarcinoma cells stained positively for p53 [10], but did not express either ER or PR $[1,10]$. This has led to the proposal that some cases with malignant transformation of adenomyosis develop de novo. Koshiyama et al reported only four cases of type II carcinomas in 564 patients $(0.74 \%)$ operated on between 1981 and 2001 [16]. Three of the four cases reported (grade 3 endometrioid adenocarcinoma, clear-cell adenocarcinoma, and serous adenocarcinoma) were of histologically unfavorable subtype [16].

It is usually difficult to make a diagnosis of type II endometrioid adenocarcinoma; a delay is often caused because of the absence of lesion in nomotopic endometrium. According to the review of Boes et al, in almost all the cases where endometrial cytology was performed, the result was negative [17]. It is usually until after the tumor has grown so much as to involve the endometrium, causing abnormal uterine bleeding or spreading outside of the uterus, a correct diagnosis is made [17]. The management of this clinical entity consists of surgery and chemotherapy.

In our own case, there was a past history of adenomyosis for 4 years. The tumor must have originated from adenomyosis because the carcinoma located only in the muscle layer of the uterus and apart from the endometrium. The endometrium was intact and cancer nests adjacent to the adenomyotic foci were observed. There is no hyperplastic or atypical changes between cancer nests and adenomyotic foci. The tumor had rapidly grown and affected many organs, killing the patient at 3 months after the appearance of a symptom.

\section{Conclusions}

Malignant transformation of adenomyosis in premenopausal women is extremely rare, and the details remain unclear including the convalescence. The case we encountered was a high-potential malignant tumor: the tumor rapidly grew and 

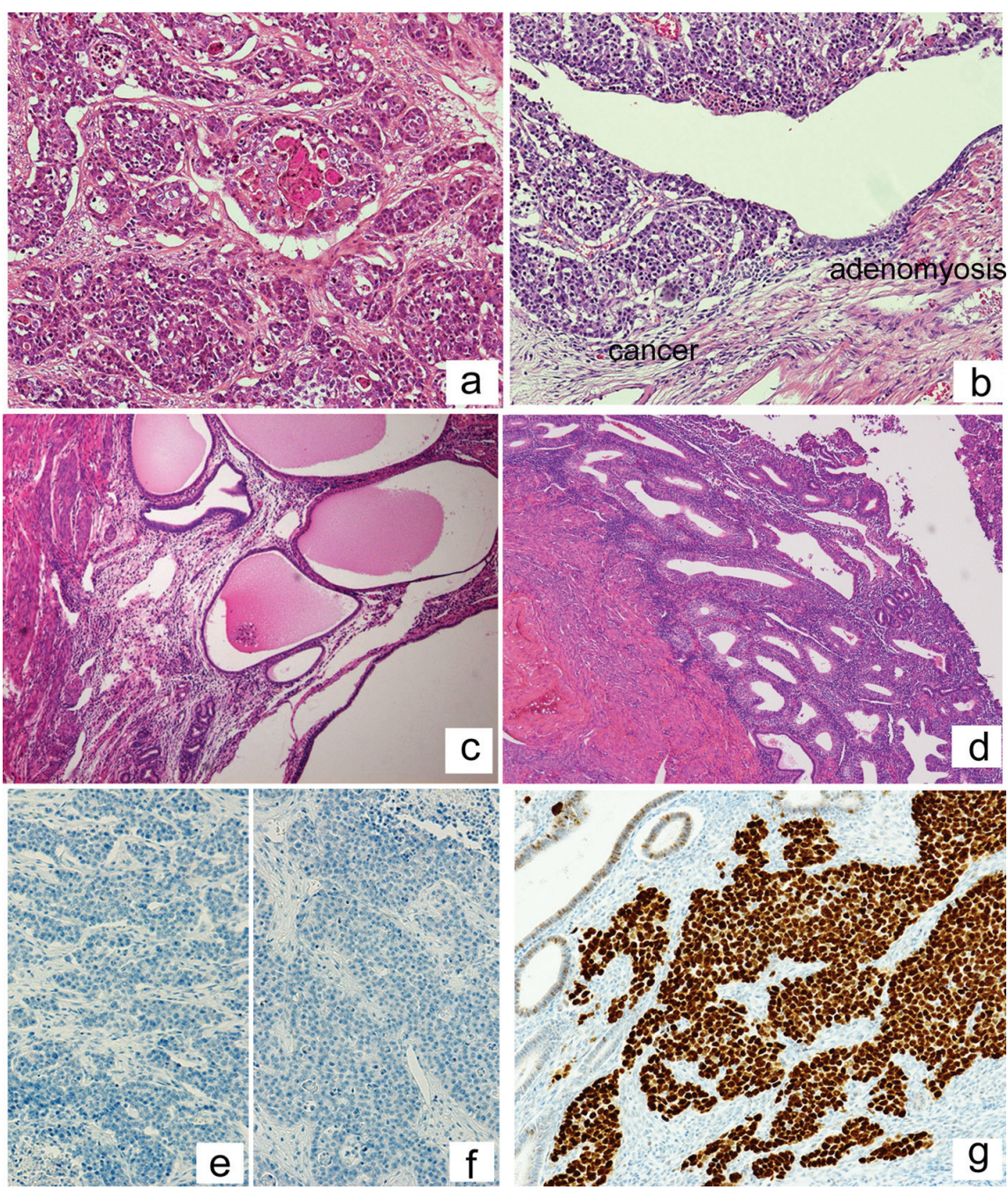

Figure 3. Histopathological analysis of the uterine specimen. Poorly differentiated endometrioid adenocarcinoma with squamous differentiation was within uterine myometrium (hematoxylin-eosin (H\&E), original magnification $\times 20)(a)$. Cancer nests adjacent to the adenomyotic foci were observed $(\mathrm{H} \& \mathrm{E}, \times 20)(\mathrm{b})$. Adenomyosis was seen in myometrium $(\mathrm{H} \& \mathrm{E}, \times 10)(\mathrm{c})$. The endometrium was unremarkable $(\mathrm{H} \& \mathrm{E}, \times 20)(\mathrm{d})$. Immunohistochemical examination: tumor cells stained positively for p53 (g), but did not express either ER (e) or PR (f).

infiltrated multiple organs in a short period, suggesting it was a type II carcinoma with biologically aggressive behavior.

\section{Acknowledgement}

We thank Kensuke Tokaichi for the editorial language.

\section{Conflict of Interests}

The authors declare that they have no conflict of interests.

\section{References}

1. Kucera E, Hejda V, Dankovcik R, Valha P, Dudas M, Fey- ereisl J. Malignant changes in adenomyosis in patients with endometrioid adenocarcinoma. Eur J Gynaecol Oncol. 2011;32(2):182-184.

2. Ferenczy A. Pathophysiology of adenomyosis. Hum Reprod Update. 1998;4(4):312-322.

3. Chen YJ, Li HY, Huang CH, Twu NF, Yen MS, Wang $\mathrm{PH}$, Chou TY, et al. Oestrogen-induced epithelial-mesenchymal transition of endometrial epithelial cells contributes to the development of adenomyosis. J Pathol. 2010;222(3):261-270.

4. Zhou S, Yi T, Liu R, Bian C, Qi X, He X, Wang K, et al. Proteomics identification of annexin A2 as a key mediator in the metastasis and proangiogenesis of endometrial cells in human adenomyosis. Mol Cell Proteomics. 2012;11(7):M112 017988.

5. Mechsner S, Bartley J, Infanger M, Loddenkemper C, Herbel J, Ebert AD. Clinical management and immuno- 
histochemical analysis of umbilical endometriosis. Arch Gynecol Obstet. 2009;280(2):235-242.

6. van Kaam KJ, Schouten JP, Nap AW, Dunselman GA, Groothuis PG. Fibromuscular differentiation in deeply infiltrating endometriosis is a reaction of resident fibroblasts to the presence of ectopic endometrium. Hum Reprod. 2008;23(12):2692-2700.

7. Leyendecker G, Wildt L, Mall G. The pathophysiology of endometriosis and adenomyosis: tissue injury and repair. Arch Gynecol Obstet. 2009;280(4):529-538.

8. Benagiano G, Brosens I, Carrara S. Adenomyosis: new knowledge is generating new treatment strategies. Womens Health (Lond Engl). 2009;5(3):297-311.

9. Greaves P, White IN. Experimental adenomyosis. Best Pract Res Clin Obstet Gynaecol. 2006;20(4):503-510.

10. Motohara K, Tashiro H, Ohtake H, Saito F, Ohba T, Katabuchi H. Endometrioid adenocarcinoma arising in adenomyosis: elucidation by periodic magnetic resonance imaging evaluations. Int J Clin Oncol. 2008;13(3):266270.

11. Koike N, Tsunemi T, Uekuri C, Akasaka J, Ito F, Shigemitsu A, Kobayashi H. Pathogenesis and malignant transformation of adenomyosis (review). Oncol Rep.
2013;29(3):861-867.

12. Kazandi M, Zeybek B, Terek MC, Zekioglu O, Ozdemir $\mathrm{N}$, Oztekin K. Grade 2 endometrioid adenocarcinoma arising from adenomyosis of the uterus: report of a case. Eur J Gynaecol Oncol. 2010;31(6):719-721.

13. Colman HI, Rosenthal AH. Carcinoma developing in areas of adenomyosis. Obstet Gynecol. 1959;14:342-348.

14. Sampson JA. Endometrial carcinoma of the ovary arising in endometrial tissue in that organ. American Journal of Obstetricsand Gynecology. 1925;9(1):111-114.

15. Abushahin N, Zhang T, Chiang S, Zhang X, Hatch K, Zheng W. Serous endometrial intraepithelial carcinoma arising in adenomyosis: a report of 5 cases. Int J Gynecol Pathol. 2011;30(3):271-281.

16. Koshiyama M, Suzuki A, Ozawa M, Fujita K, Sakakibara A, Kawamura M, Takahashi S, et al. Adenocarcinomas arising from uterine adenomyosis: a report of four cases. Int J Gynecol Pathol. 2002;21(3):239-245.

17. Boes AS, Tousseyn T, Vandenput I, Timmerman D, Vergote I, Moerman P, Amant F. Pitfall in the diagnosis of endometrial cancer: case report of an endometrioid adenocarcinoma arising from uterine adenomyosis. Eur J Gynaecol Oncol. 2011;32(4):431-434. 